\title{
Transanal total mesorectal excision for low rectal cancer: a case-matched study comparing TaTME versus standard laparoscopic TME
}

This article was published in the following Dove Press journal: Cancer Management and Research

\author{
Mateusz Rubinkiewicz' \\ Michał Nowakowski \\ Mateusz Wierdak ${ }^{1,3}$ \\ Magdalena Mizera' \\ Marcin Dembiński' \\ Magdalena Pisarska ${ }^{1,3}$ \\ Piotr Major ${ }^{1,3}$ \\ Piotr Małczak ${ }^{1,3}$ \\ Andrzej Budzyński',3 \\ Michał Pędziwiatr ${ }^{1,3}$ \\ '2nd Department of General Surgery, \\ Jagiellonian University Medical \\ College, Krakow, Poland; ${ }^{2}$ Department \\ of Medical Education, Jagiellonian \\ University Medical College, Krakow, \\ Poland; ${ }^{3}$ Centre for Research, Training \\ and Innovation in Surgery (CERTAIN \\ Surgery), Krakow, Poland
}

Correspondence: Michał Pędziwiatr 2nd Department of General Surgery, Jagiellonian University Medical College, Kopernika 2I, Kraków 31-50I, Poland Tel +48 608552323

$\mathrm{Fax}+48 \quad$ I2 42I 3456

Email michal.pedziwiatr@uj.edu.pl
Background: Transanal total mesorectal excision (TaTME) is emerging as a novel alternative to laparoscopic total mesorectal excision (LaTME). The aim of this study was to compare clinical and pathological results from these two techniques in patients undergoing rectal resections because of low rectal cancer.

Materials and methods: Thirty-five patients undergoing TaTME were matched with 35 patients operated on using LaTME. Composite primary endpoint (complete TME, negative circumferential resection margin [pCRM], and distal resection margin [pDRM]) was used to assess pathological quality specimens. Secondary outcomes included operative and postoperative parameters (operative time, total blood loss, postoperative morbidity, length of stay, 30-day mortality).

Results: Composite primary endpoint was achieved by $85 \%$ of subjects in the TaTME group and $82 \%$ of subjects in the LaTME group $(P=0.66)$. Mean pCRM was $1.1 \pm 1.29$ vs $0.99 \pm 0.78$ $\mathrm{mm}(P=0.25)$. Distal pDRM was $1.57 \pm 0.92$ and $1.98 \pm 1.22 \mathrm{~cm}(P=0.15)$. In the TaTME and LaTME groups, respectively, complete mesorectal excision was achieved in $89 \%$ and $83 \%$ of subjects, while excision was nearly complete for the remaining $11 \%$ and $17 \%(P=0.23)$.

Conclusion: TaTME appears to be a noninferior alternative to laparoscopic surgery. TaTME allows for quality retrieval of surgical specimens with comparable clinical outcomes with LaTME. Keywords: transanal TME, laparoscopic TME, rectal cancer, low rectal cancer, minimally invasive surgery

\section{Background}

Treatment results in rectal cancer patients depend strongly on the quality of surgery; therefore, successful oncologic resection requires complete mesorectal excision without injury to the mesorectal fascia and with preservation of adequate distal and radial margins and adequate lymphadenectomy. ${ }^{1-4}$ Regardless of the surgical access method, the procedure itself is challenging (particularly in obese male patients), requires substantial expertise, and final outcomes in the global patient population are suboptimal. ${ }^{5,6}$ The oncological safety of laparoscopic surgery has been proven in randomized control trials (RCTs); therefore, it could be treated as a reference as well as an open approach. ${ }^{7,8}$ Laparoscopic surgery of the rectum, which has been performed for more than two decades, has reached a similar level of efficacy and is regarded, at the minimum, as noninferior to open operation. ${ }^{8-13}$ Both techniques - open and laparoscopic - have been standardized and are considered equivalent. However, they may have reached their maximum levels of efficacy, and a substantive improvement in treatment results through refinement of the existing methods is unlikely. This plateau, 
observed for several years, has prompted a search for more effective methods to increase the quality of mesorectal excision. By application of the perineal approach in intersphinteric resection, Rullier pioneered sphincter-saving surgery in low rectal tumors. ${ }^{14}$ Expectations have recently risen with introduction of the transanal (or hybrid) approach, referred to as transanal total mesorectal excision (TaTME). ${ }^{15}$ The method itself appears highly promising, particularly for patients with cancer located in the lower rectum, where technical difficulties are the most severe, and the risk of permanent stoma is highest. ${ }^{16}$ TaTME is also expected to diminish the conversion rate and risk of specimen perforation. Moreover, it was recently reported that quality pathological specimens have been retrieved following TaTME. ${ }^{17-20}$ However, full evaluation of this technique requires longlasting, well-designed, and properly performed prospective trials. Unfortunately, ongoing studies are not expected to be completed for several years. ${ }^{5}$ This prompted us to undertake a case-matched study based on our own experience, to compare laparoscopic TME (LaTME) and TaTME with regard to the surgical quality of low rectal cancer resection. Aim of the study is to compare short-term clinical and pathological outcomes of TaTME and LaTME in low rectal cancer.

\section{Materials and methods Setting and design}

All procedures were performed in a tertiary referral university hospital participating in the COLOR III RCT. The annual volume in our unit is around 120 colorectal procedures, 50 of which involve rectal resections. The TaTME procedure was implemented in 2014. Patients with cancer of the lower rectum $(<5 \mathrm{~cm}$ from the anal verge) and who underwent surgery via TaTME were included in group 1. Patients from the TaTME group were matched with patients who underwent LaTME (group 2). Matching was done by an independent administrative officer, for age, body mass index (BMI), sex, distance of the tumor from the anal verge, and stage of the disease. To avoid potential bias, the officer was not made aware of the study concept. All surgeons in our team with expert skills in laparoscopic surgery participated in a hands-on cadaver TaTME course before performing the TaTME procedure on our patients. The first 10 TaTME cases performed at our institution were excluded from the analysis to reduce the bias during the learning curve for the operation. The same experienced pathologist assesses all specimens in accordance with a classification derived by Quirke et al. ${ }^{21,22}$ Nearly complete mesorectal excision was defined as defects $<5 \mathrm{~mm}$ in the mesorectal fascia. R1 resection margin was defined as a radial or distal margin $<1$ $\mathrm{mm}$. Complete mesorectal excision was defined as no defects in the mesorectal fascia.

\section{Patients}

Prospective collection of data from patients undergoing TaTME surgery for low ( $<5 \mathrm{~cm}$ from the anal verge) rectal adenocarcinoma was performed between January 2015 and January 2018. A control LaTME group was from 2012 to 2014, when LaTME was a standard procedure for treating low rectal cancer at our clinic. The protocol of treatment, including chemoradiotherapy and perioperative care, did not change during the study duration. Patients initially admitted for abdominoperineal resection and end colostomy were excluded from the analysis. Patients included in the group were not participants in the COLOR III trial. All procedures were performed by one of surgeons with expert skills in colorectal surgery procedures. Patients were submitted for preoperative chemoradiotherapy when clinically indicated (T3 or positive lymph nodes in preoperative MRI staging). We routinely use a long course of chemoradiotherapy, which consists of 50.4 Gy combined with chemotherapy followed by surgery after 8 weeks. Preopretive chemoradiotherapy was indicated in 31 patients in each group. In LaTME group, 18 patients had cT3 stage alone as an indication for radiotherapy, 6 had cT3N+, 6 had cT2N+, and $1 \mathrm{cT} 1 \mathrm{~N}+$. In TaTME group, 17 patients had cT3 alone, 6 had cT3N+, 7 had cT2N+, and 1 had cT1N+. Four patients in LaTME and five patients in TaTME had $<2 \mathrm{~mm}$ magnetic resonance imaging circumferencial resection margin (circumferential resection margin [pCRM]); they were reevaluated before the surgery. In these patients, we managed to achieve downstaging before the surgery; thus there were no suspicion of mesorectal fascia infiltration. Perioperatively, all patients were treated in accordance with the Enhanced Recovery after Surgery (ERAS) protocol, which is a standard for care at our institution. ${ }^{23-25}$

\section{Measured outcomes}

The primary outcome was a composite endpoint defined as negative pCRM, negative distal resection margin (pDRM), and complete mesorectal excision. Secondary outcomes were operative and postoperative parameters (operative time, total blood loss, postoperative morbidity, length of stay, 30-day mortality). Postoperative morbidity was assessed using Clavien-Dindo classification. ${ }^{26}$ Serious complications were defined as Clavien-Dindo III-V.

\section{Operative technique}

The operative technique used is detailed elsewhere. ${ }^{27} \mathrm{We}$ routinely use a Karl Storz TEO ${ }^{\circledR}$ platform as an optimization 
of the original technique. ${ }^{17}$ The procedure is performed using a one-team approach. The anastomosis is facilitated with a circular stapler, and in selected cases (when internal sphincter resection was needed to gain negative margin or stapler anastomosis was not possible), coloanal anastomosis was performed. No additional procedures (ie, coloplasty) were performed within anastomosis. Full mobilization of the splenic flexure was done to facilitate tension-free anastomosis. Defunctioning ileostomy is created routinely.

\section{Statistical analysis and ethical approval}

All data were analyzed using Statistica version 13.0 PL (StatSoft Inc., Tulsa, OK, USA). The results are presented as mean and SD, median, and IQR. The chi-squared test of independence was used for evaluating categorical variables. The Shapiro-Wilk test was used to check for normal distribution of data and Student's $t$-test was used for normally distributed quantitative data. The Mann-Whitney $U$ test was used for non-normally distributed quantitative variables. Statistical significance was set at $P<0.05$.

All procedures were performed in accordance with the ethical standards of the 1964 Declaration of Helsinki and its later amendments (Fortaleza 2013). The study was approved by the Local Ethics Committee of the Jagiellonian University Medical College. Every patient signed an informed consent prior to inclusion in the study.

\section{Results}

Table 1 presents demographical characteristics of the study group. Composite primary endpoint was reached by $86 \%$ of the subjects in the TaTME group and $83 \%$ in the LaTME group $(P=0.74)$. Table 2 summarizes the results.

Patients undergoing TaTME resection had a wider radial margin compared with those undergoing standard laparos- copy, although the data did not reach statistical significance ( $1.41 \pm 1.29$ vs $0.99 \pm 0.78 \mathrm{~mm} ; P=0.25$ ). The distal margin was shorter in the TaTME group than the laparoscopy group, but again did not reach statistical significance $(1.57 \pm 0.92$ vs $1.98 \pm 1.22 \mathrm{~cm} ; P=0.15$ ).

When using the TaTME technique, complete mesorectal excision was achieved in $89 \%$ of patients, and in $11 \%$, excision was nearly complete. For LaTME, $83 \%$ had complete mesorectal excision and $17 \%$ nearly complete resection. There were no cases of incomplete mesorectal excision.

Operative time in the TaTME group was significantly longer and reached a mean of $271 \pm 63$ minutes, whereas in the LaTME group, the mean time was $219 \pm 45$ minutes $(P=0.001)$. Mean blood loss was $165 \pm 148 \mathrm{~mL}$ in the TaTME group and $113 \pm 75 \mathrm{~mL}$ in the LaTME group $(P=0.07)$. No conversions to an open approach were needed in either group. There were two intraoperative complications in the LaTME group (one bowel wall perforation with intestinal content spillage and one anastomotic leakage identified during leak test and that was resolved by adding additional sutures) and four in the TaTME group (two anastomotic leakages identified intraoperatively, which were solved by adding additional sutures in transanal approach and four purse-string failures, although none of these patients had subsequent abdominal abscess in the postoperative course). We managed to perform primary anastomosis in all patients. Six patients in the TaTME group and eight in the LaTME group suffered from postoperative complications $(17 \%$ vs $23 \% ; P=0.21$ ). Three serious complications occurred in the TaTME group and four in the LaTME group (9\% vs $12 \% ; P=0.80)$. We had to create end colostomy in one of our patients in the TaTME group as a result of anastomotic leakage with septic complications after failed EndoVac ${ }^{\circledR}$ management. There were no differences for mean length of hospital stay ( $9.7 \pm 10.8$ vs $8.8 \pm 7.8$ days; $P=0.71)$. No 30 -day

Table I Demographical characteristics

\begin{tabular}{|c|c|c|c|}
\hline & LaTME & TaTME & $P$-value \\
\hline Number of patients & 35 & 35 & \\
\hline Age & $60.3 \pm 10.2$ & $64.3 \pm 10.1$ & 0.08 \\
\hline Sex (women) & $\mathrm{II}(3 \mathrm{I} \%)$ & $\mathrm{II}(3 \mathrm{I} \%)$ & 1.00 \\
\hline Body mass index $\left(\mathrm{kg} / \mathrm{m}^{2}\right)$ & $27.1 \pm 4.71$ & $26.1 \pm 4.09$ & 0.22 \\
\hline Distance of the tumor from the anal verge $(\mathrm{cm})$ & $3.19 \pm 1.47$ (range I-5) & $2.9 \pm 1.17$ (range I-5) & 0.47 \\
\hline ASA & \begin{tabular}{|l|} 
I-6 \\
II-20 \\
III-9 \\
\end{tabular} & $\begin{array}{l}1-8 \\
\text { II-19 } \\
\text { III-8 }\end{array}$ & 0.60 \\
\hline Preoperative neoadjuvant treatment (yes/no) & $31 / 4$ & $3 I / 4$ & $\mathrm{I}$ \\
\hline Type of anastomosis (stapled/hand-sewn) & $29 / 6$ & $30 / 5$ & 0.74 \\
\hline
\end{tabular}

Abbreviations: ASA, American Society of Anesthesiologists Classification; LaTME, laparoscopic total mesorectal excision; TaTME, transanal total mesorectal excision. 
Table 2 Number of complications

\begin{tabular}{|c|c|c|c|c|}
\hline Clavien-Dindo grade & LaTME & $\mathbf{N}(\%)$ & TaTME & $\mathbf{N}(\%)$ \\
\hline V & & & & 0 \\
\hline IV & & & $\begin{array}{l}\text { Anastomotic leakage with concomitant } \\
\text { sepsis (operative treatment) }\end{array}$ & I (3) \\
\hline IIIlb & $\begin{array}{l}\text { Anastomotic leakage (operative } \\
\text { treatment) } \\
\text { Postoperative ileus (operative treatment) }\end{array}$ & $\begin{array}{l}2(6) \\
1(3)\end{array}$ & Anastomotic leakage & $2(6)$ \\
\hline IIIla & $\begin{array}{l}\text { Anastomotic leakage (percutaneus } \\
\text { drainage of the near-anastomotic } \\
\text { abscess) }\end{array}$ & I (3) & & 0 \\
\hline II & $\begin{array}{l}\text { High output stoma } \\
\text { Anastomotic leakage (conservative } \\
\text { treatment) }\end{array}$ & $\begin{array}{l}\text { I (3) } \\
2(6)\end{array}$ & $\begin{array}{l}\text { Postoperative ileus (conservative } \\
\text { treatment) }\end{array}$ & $2(6)$ \\
\hline I & Postoperative fever of unknown origin & I (3) & $\begin{array}{l}\text { Radial nerve paresis (due to prolonged } \\
\text { compression on the operating table) }\end{array}$ & I (3) \\
\hline Total & & $8(23)$ & & $6(17)$ \\
\hline
\end{tabular}

Abbreviations: LaTME, laparoscopic total mesorectal excision; TaTME, transanal total mesorectal excision.

mortality was observed in either group. Table 3 presents a summary of the complications.

\section{Discussion}

In our study, we showed TaTME is safe and feasible, providing similar quality of resected oncological specimens. We managed to achieve acceptable resection margins, as well as intact mesorectal fascia using both the laparoscopic and transanal approach.

One of the greatest drawbacks of the laparoscopic approach is the quality of the pathological specimens. In the study, we chose to set a composite endpoint assessing the pCRM, pDRM, and completeness of mesorectal excision together. Although pCRM is commonly considered the most important aspect for quality assessment, other components should not be underestimated. Abbas et $\mathrm{al}^{28}$ reported that, with the laparoscopic technique, there is a risk of a smaller pCRM, which may be inadequate for achieving a satisfactory oncological outcome. ${ }^{29}$ Additionally, large-scale RCTs such as ACOSOG and ALACART failed to demonstrate noninferiority for laparoscopic surgery compared with an open approach. ${ }^{30}$

In the present study, with the TaTME technique, we obtained similar results to those from LaTME for resection margins, as well as pathological specimen quality. Perdawood et al reported on a series of 100 TaTME procedures in which they obtained results comparable with those of laparoscopic and open surgery for pCRM. ${ }^{18}$ Other authors have described similar results. ${ }^{19,31}$ In contrast, an initial meta-analysis of seven studies revealed better $\mathrm{pCRM}$ and lower positive $\mathrm{pCRM}$ in patients who underwent transanal technique surgeries.
However, it used a fixed effects model for calculations, which may have biased the results in studies with high heterogeneity. ${ }^{32}$ Of note, $\mathrm{pCRM}<1 \mathrm{~mm}$ is a risk factor for locoregional recurrence, as well as distant metastasis, and pCRM $>1$ and $<5 \mathrm{~mm}$ reduced the amount of locoregional recurrence, but not distant metastases. Only pCRM $>5 \mathrm{~mm}$ facilitated diminished incidence of both distant and locoregional recurrence. ${ }^{33} \mathrm{pCRM}<1 \mathrm{~mm}$ is also a negative prognostic factor for overall survival. ${ }^{34}$ In our patients, both techniques allowed for achievement of an optimal pCRM wider than $5 \mathrm{~mm}$. Using TaTME, we were able to reach a pCRM that averaged $5 \mathrm{~mm}$ wider than when using the laparoscopic technique. Nonetheless, the data did not reach statistical significance, likely because of the relatively small study group size.

Another important factor in rectal cancer treatment is the completeness of the mesorectal excision. Intact rectal fascia diminishes the incidence of recurrence. ${ }^{21}$ Creavin et al reported in their meta-analysis that superficial damage of the mesorectal fascia is more common in laparoscopic surgery compared with the open approach. ${ }^{35}$ Additionally, total mesorectal excision, defined as an intact mesorectum, negative pCRM, and distal margin, was achieved more often when using open surgery. ${ }^{35}$ Of note, an acceptable mesorectum, defined as intact or superficial defects of the fascia, was achieved in both approaches at the same rate. ${ }^{35}$ However, that meta-analysis included only RCTs published in 2010 and 2015. Moreover, another meta-analysis covering 1966 through 2016, by Pędziwiatr et al, revealed no differences between the open and laparoscopic techniques regarding quality of surgical specimens and short- and long-term oncological outcomes. ${ }^{8}$ In our opinion, owing to better lower 
Table 3 Outcomes assessment

\begin{tabular}{|c|c|c|c|}
\hline & LaTME & TaTME & $P$-value \\
\hline \multicolumn{4}{|l|}{ Primary outcome } \\
\hline $\begin{array}{l}n(\%) \text { of patients with negative pCRM and } \\
\text { pDRM and complete mesorectal excision }\end{array}$ & $29 / 35(83 \%)$ & $30 / 35(86 \%)$ & 0.74 \\
\hline \multicolumn{4}{|l|}{ Primary outcome components } \\
\hline $\mathrm{PCRM} \geq \mathrm{I} \mathrm{mm}$ & $35 / 35(100 \%)$ & $34 / 35(97 \%)$ & 0.25 \\
\hline $\mathrm{pDRM} \geq \mathrm{I} \mathrm{mm}$ & $34 / 35(97 \%)$ & $35 / 35(100 \%)$ & 0.25 \\
\hline $\begin{array}{l}\text { Total mesorectal excision, } \mathrm{n}(\%) \\
\text { Complete } \\
\text { Nearly complete } \\
\text { Incomplete }\end{array}$ & $\begin{array}{l}29 / 35(83) \\
6 / 35(17) \\
0 / 35(0)\end{array}$ & $\begin{array}{l}31 / 35(89) \\
4 / 35(1 \mathrm{I}) \\
0 / 35(0)\end{array}$ & 0.23 \\
\hline \multicolumn{4}{|l|}{ Secondary outcomes } \\
\hline Surgery duration (mean) & $219 \pm 45$ minutes & $27 I \pm 63$ minutes & 0.00 \\
\hline Blood loss (mean) & $\mathrm{I} / 3 \pm 75 \mathrm{~mL}$ & $165 \pm 148 \mathrm{~mL}$ & 0.07 \\
\hline Postoperative complications & $8(22 \%)$ & $6(17 \%)$ & 0.55 \\
\hline Serious complications (CD 3-5) & $4(11 \%)$ & $3(9 \%)$ & 0.69 \\
\hline AJCC stage & $\begin{array}{l}0-7 \\
1-7 \\
2-10 \\
3-11 \\
4-0\end{array}$ & $\begin{array}{l}0-8 \\
1-3 \\
2-10 \\
3-14 \\
4-0\end{array}$ & 0.58 \\
\hline \multicolumn{4}{|l|}{ Pathological TNM staging } \\
\hline $\begin{array}{l}\mathrm{PT} \\
0 \\
1 \\
2 \\
3 \\
4 \\
\end{array}$ & $\begin{array}{l}7 \\
6 \\
3 \\
19 \\
0 \\
\end{array}$ & $\begin{array}{l}8 \\
5 \\
3 \\
19 \\
0 \\
\end{array}$ & 0.93 \\
\hline $\begin{array}{l}\mathrm{pN} \\
0 \\
1 \\
2\end{array}$ & $\begin{array}{l}24 \\
6 \\
4\end{array}$ & $\begin{array}{l}21 \\
8 \\
5\end{array}$ & 0.58 \\
\hline
\end{tabular}

Abbreviations: AJCC, American Joint Committee on Cancer; LaTME, laparoscopic total mesorectal excision; pCRM, circumferential resection margin; pDRM, distal resection margin; TaTME, transanal total mesorectal excision.

pelvic insight and visualization of the distal margin, TaTME allows the surgeon to obtain higher-quality specimens than does pure laparoscopy. Nevertheless, we do feel TaTME pushes the surgeon to seek wider margins to achieve better specimen quality; this could lead to dangerous complications such as nerve injuries or damage to surrounding organs. ${ }^{36,37}$

TaTME initially appears to be a comparable method with classic laparoscopy in terms of clinical outcomes, with the only difference being longer operating time in TaTME. . $^{17,38,39}$ This burden may be resolved by using a two-team approach: two laparoscopic sets and two teams of surgeons, wherein one team is responsible for the transanal step and the other for the abdominal. The two-team approach is promising when viewed in comparison with the one-team approach. Lacy et al achieved an average operation time of 166 minutes using two teams, whereas Caycedo-Marulanda, using a one-team approach, averaged 283 minutes. ${ }^{17,40}$ However, a two-team approach requires more personnel and more surgical equipment, which may be limiting factors in some units.

In our material, we had four cases of purse-string suture failure, which can lead to contamination of the operating field. ${ }^{41}$ None of those patients had bowel content spillage - a related complication as an abdominal abscess. Other complications were unrelated to the technique included anastomotic failure and bowel wall perforation, which were repaired intraoperatively. Other potential intraoperative difficulties are problems with correct dissection plane identification, maintaining stable peritoneum, and adjacent organ injury such as urethra or hypogastric plexus. ${ }^{42}$

The long-accepted advantages of minimally invasive techniques include shorter hospitalization time and lower morbidity. Importantly, though older patients in particular benefit from laparoscopic approaches. Minimalizing perioperative trauma in this group of patients is crucial 
because of increased comorbidities. The laparoscopic approach lowers not only perioperative complication rates but also overall mortality. ${ }^{43}$ Additionally, a combination of minimally invasive techniques with the ERAS protocol allows for further improvement in recovery and outcomes..$^{44,45}$

Our study has several clear limitations. First, the study group is small, which could increase the chance for type II errors; thus, it is obviously underpowered. However, to prove the advance of the TaTME technique, a considerably large group is required, as was calculated by the COLOR III designers. A group of 732 cases with randomization of 2:1 is needed to demonstrate the superiority of a new technique over LaTME. ${ }^{5}$ It would take a great deal of time to collect such a large amount of cases; therefore, only an RCT may deliver substantive evidence. Moreover, TaTME is a new technique that requires a specific procedural surgeon and institutional experience. ${ }^{46,47}$ Koedam et al calculated that at least 40 cases are required for a surgeon to become proficient. ${ }^{46}$ After exclusion of the first 10 cases, our group had 34 participants; however, we analyzed patients only with low rectal cancer, and cases operated because of mid rectal cancer (5-10 cm from the anal verge) were also excluded. We realize that including patients during the learning curve period may bias the outcomes. The resolution to this problem is multicenter RCTs with external-quality auditing. RCTs comparing laparoscopic and TaTME have already begun. In 2016, COLOR III was launched. This is a well-designed and properly sampled trial with endpoints focusing on long-term outcomes. ${ }^{5}$ Our department participates in this. Other trials were also launched, such as ETAP-GRECCAR 11 and another designed by the TAU-TEM study group. ${ }^{48,49}$ Those studies' results will be published over the next several years; therefore, until that time, we need to rely on data from smaller trials of possibly lower quality, which nevertheless provide valuable insight on the topic, with relatively low risk of bias. The other limitation is that the study was designed as case-matched. We were unable to find perfect matches; therefore, the patients in TaTME group were older than in LaTME group. We also realize that in such a small group of patients, this may create bias. BMI in the LaTME group was also slightly higher than in the TaTME group, which was also a result of imperfect matching, though the difference was small and not statistically significant.

\section{Conclusion}

TaTME appears to be noninferior to laparoscopic surgery, although the current data are still insufficient to fully validate the technique. TaTME provides surgeons an option for qual- ity retrieval of surgical specimens with comparable clinical outcomes with LaTME. However, data from large RCTs are still necessary to confirm this method as a gold standard in rectal cancer surgery.

\section{Disclosure}

The authors report no conflicts of interest in this work.

\section{References}

1. Langman G, Loughrey M, Shepherd N, Quirke P. Association of Coloproctology of Great Britain \& Ireland (ACPGBI): Guidelines for the Management of Cancer of the Colon, Rectum and Anus (2017) - Pathology Standards and Datasets. Colorectal Dis. 2017;19(Suppl 1):74-81.

2. West NP, Morris EJ, Rotimi O, Cairns A, Finan PJ, Quirke P. Pathology grading of colon cancer surgical resection and its association with survival: a retrospective observational study. Lancet Oncol. 2008;9(9):857-865.

3. Daniels IR, Fisher SE, Heald RJ, Moran BJ. Accurate staging, selective preoperative therapy and optimal surgery improves outcome in rectal cancer: a review of the recent evidence. Colorectal Dis. 2007;9(4):290-301.

4. Cecil TD, Sexton R, Moran BJ, Heald RJ. Total mesorectal excision results in low local recurrence rates in lymph node-positive rectal cancer. Dis Colon Rectum. 2004;47(7):1145-1149; discussion 1149-1150.

5. Deijen CL, Velthuis S, Tsai A, et al. COLOR III: a multicentre randomised clinical trial comparing transanal TME versus laparoscopic TME for mid and low rectal cancer. Surg Endosc. 2016;30(8):3210-3215.

6. Jamali FR, Soweid AM, Dimassi H, Bailey C, Leroy J, Marescaux J. Evaluating the degree of difficulty of laparoscopic colorectal surgery. Arch Surg. 2008;143(8):762-767; discussion 768.

7. Bonjer HJ, Deijen CL, Abis GA, et al; COLOR II Study Group. A randomized trial of laparoscopic versus open surgery for rectal cancer. N Engl J Med. 2015;372(14):1324-1332.

8. Pędziwiatr M, Małczak P, Mizera M, et al. There is no difference in outcome between laparoscopic and open surgery for rectal cancer: a systematic review and meta-analysis on short- and long-term oncologic outcomes. Tech Coloproctol. 2017;21(8):595-604.

9. de'Angelis N, Landi F, Vitali GC, et al. Multicentre propensity scorematched analysis of laparoscopic versus open surgery for T4 rectal cancer. Surg Endosc. 2017;31(8):3106-3121.

10. Kim IY, Kim BR, Kim HS, Kim YW. Differences in clinical features between laparoscopy and open resection for primary tumor in patients with stage IV colorectal cancer. Onco Targets Ther. 2015;8:3441-3448.

11. Nussbaum DP, Speicher PJ, Ganapathi AM, et al. Laparoscopic versus open low anterior resection for rectal cancer: results from the national cancer data base. J Gastrointest Surg. 2015;19(1):124-13 ; discussion 131-132.

12. Dušek T, Ferko A, Orhalmi J, et al. Rectal cancer within $10 \mathrm{~cm}$. Comparison of the radicality of laparoscopic and open surgical techniques with regard to the circumferential resection margin and the completeness of mesorectal excision. Rozhl Chir. 2013;92(6):312-319.

13. Hida K, Okamura R, Sakai Y, et al; Japan Society of Laparoscopic Colorectal Surgery. Open versus laparoscopic surgery for advanced low rectal cancer: a large, multicenter, propensity score matched cohort study in Japan. Ann Surg. 2018;268(2):318-324.

14. Rullier E, Zerbib F, Laurent C, et al. Intersphincteric resection with excision of internal anal sphincter for conservative treatment of very low rectal cancer. Dis Colon Rectum. 1999;42(9):1168-1175.

15. Lacy AM, Adelsdorfer C. Totally transrectal endoscopic total mesorectal excision (TME). Colorectal Dis. 2011;13(Suppl 7):43-46.

16. Yoo RN, Kim G, Kye BH, Cho HM, Kim H. The fate of preserved sphincter in rectal cancer patients. Int J Colorectal Dis. 2018;33(6):745-753.

17. Lacy AM, Tasende MM, Delgado S, et al. Transanal total mesorectal excision for rectal cancer: outcomes after 140 patients. J Am Coll Surg. $2015 ; 221(2): 415-423$. 
18. Perdawood SK, Thinggaard BS, Bjoern MX. Effect of transanal total mesorectal excision for rectal cancer: comparison of short-term outcomes with laparoscopic and open surgeries. Surg Endosc. 2018;32(5):2312-2321.

19. Rasulov AO, Mamedli ZZ, Gordeyev SS, Kozlov NA, Dzhumabaev HE. Short-term outcomes after transanal and laparoscopic total mesorectal excision for rectal cancer. Tech Coloproctol. 2016;20(4):227-234.

20. Deijen CL, Tsai A, Koedam TW, et al. Clinical outcomes and case volume effect of transanal total mesorectal excision for rectal cancer: a systematic review. Tech Coloproctol. 2016;20(12):811-824.

21. Nagtegaal ID, van de Velde CJ, van der Worp E, et al; Cooperative Clinical Investigators of the Dutch Colorectal Cancer Group. Macroscopic evaluation of rectal cancer resection specimen: clinical significance of the pathologist in quality control. J Clin Oncol. 2002;20(7):1729-1734.

22. Quirke P, Morris E. Reporting colorectal cancer. Histopathology. 2007;50(1):103-112.

23. Pędziwiatr M, Pisarska M, Kisielewski M, et al. ERAS protocol in laparoscopic surgery for colonic versus rectal carcinoma: are there differences in short-term outcomes? Med Oncol. 2016;33(6):56.

24. Kisielewski M, Rubinkiewicz M, Pędziwiatr M, et al. Are we ready for the ERAS protocol in colorectal surgery? Wideochir Inne Tech Maloinwazyjne. 2017;12(1):7-12.

25. Pisarska M, Pędziwiatr M, Małczak P, et al. Do we really need the full compliance with ERAS protocol in laparoscopic colorectal surgery? A prospective cohort study. Int J Surg. 2016;36(Pt A):377-382.

26. Clavien PA, Barkun J, de Oliveira ML, et al. The Clavien-Dindo classification of surgical complications: five-year experience. Ann Surg. 2009;250(2):187-196.

27. Suwanabol PA, Maykel JA. Transanal total mesorectal excision: a novel approach to rectal surgery. Clin Colon Rectal Surg. 2017;30(2):120-129

28. Abbas SK, Yelika SB, You K, et al. Rectal cancer should not be resected laparoscopically: the rationale and the data. Tech Coloproctol. 2017;21:237-240.

29. Martínez-Pérez A, Carra MC, Brunetti F, de'Angelis N. Short-term clinical outcomes of laparoscopic vs open rectal excision for rectal cancer: a systematic review and meta-analysis. World J Gastroenterol. 2017;23(44):7906-7916.

30. Fleshman J, Branda M, Sargent DJ, et al. Effect of laparoscopic-assisted resection vs open resection of stage II or III rectal cancer on pathologic outcomes: the ACOSOG Z6051 randomized clinical trial. JAMA 2015;314(13):1346-1355.

31. Fernández-Hevia M, Delgado S, Castells A, et al. Transanal total mesorectal excision in rectal cancer: short-term outcomes in comparison with laparoscopic surgery. Ann Surg. 2015;261(2):221-227.

32. Jiang HP, LiYS, Wang B, et al. Pathological outcomes of transanal versus laparoscopic total mesorectal excision for rectal cancer: a systematic review with meta-analysis. Surg Endosc. 2018;32(6):2632-2642.

33. Sung S, Kim SH, Lee JH, et al. Continuous effect of radial resection margin on recurrence and survival in rectal cancer patients who receive preoperative chemoradiation and curative surgery: a multicenter retrospective analysis. Int J Radiat Oncol Biol Phys. 2017;98(3):647-653.

34. Tilney HS, Rasheed S, Northover JM, Tekkis PP. The influence of circumferential resection margins on long-term outcomes following rectal cancer surgery. Dis Colon Rectum. 2009;52(10):1723-1729.
35. Creavin B, Kelly ME, Ryan E, Winter DC. Meta-analysis of the impact of surgical approach on the grade of mesorectal excision in rectal cancer. Br J Surg. 2017;104(12):1609-1619.

36. Kim NK. Anatomic basis of sharp pelvic dissection for curative resection of rectal cancer. Yonsei Med J. 2005;46(6):737-749.

37. Shirouzu K, Ogata Y, Araki Y. Oncologic and functional results of total mesorectal excision and autonomic nerve-preserving operation for advanced lower rectal cancer. Dis Colon Rectum. 2004;47(9): 1442-1447.

38. Muratore A, Mellano A, Marsanic P, De Simone M. Transanal total mesorectal excision (taTME) for cancer located in the lower rectum: short- and mid-term results. Eur J Surg Oncol. 2015;41(4):478-483.

39. Veltcamp Helbach M, Deijen CL, Velthuis S, Bonjer HJ, Tuynman JB, Sietses C. Transanal total mesorectal excision for rectal carcinoma: short-term outcomes and experience after 80 cases. Surg Endosc. 2016;30(2):464-470.

40. Caycedo-Marulanda A, Jiang HY, Kohtakangas EL. Outcomes of a single surgeon-based transanal-total mesorectal excision (TATME) for rectal cancer. J Gastrointest Cancer. Epub 2017 Jul 13.

41. Martin-Perez B, Otero-Piñeiro A, Lacy AM. Purse-string rupture: pitfalls of transanal total mesorectal excision (Cecil approach). Tech Coloproctol. 2018;22(5):393-394.

42. Penna M, Hompes R, Arnold S, et al; TaTME Registry Collaborative. Transanal total mesorectal excision. Ann Surg. 2017;266(1):111-117.

43. Antoniou SA, Antoniou GA, Koch OO, Pointner R, Granderath FA Laparoscopic colorectal surgery confers lower mortality in the elderly: a systematic review and meta-analysis of 66,483 patients. Surg Endosc. 2015;29(2):322-333.

44. Pędziwiatr M, Wierdak M, Nowakowski et al. Cost minimization analysis of laparoscopic surgery for colorectal cancer within the enhanced recovery after surgery (ERAS) protocol: a single-centre, case-matched study. Wideochirurgia i Inne Tech Maloinwazyjn. 2016;11:14-21.

45. Pędziwiatr M, Kisialeuski M, Wierdak M, et al. Early implementation of Enhanced Recovery after Surgery (ERAS $®)$ protocol - compliance improves outcomes: a prospective cohort study. Int J Surg. 2015;21:75-81.

46. Koedam TWA, Veltcamp Helbach M, van de Ven PM, et al. Transanal total mesorectal excision for rectal cancer: evaluation of the learning curve. Tech Coloproctol. 2018;22(4):279-287.

47. Adamina M, Buchs NC, Penna M, Hompes R; St. Gallen Colorectal Consensus Expert Group. St.Gallen consensus on safe implementation of transanal total mesorectal excision. Surg Endosc. 2018;32(3):1091-1103.

48. Lelong B, de Chaisemartin C, Meillat H, et al; French Research Group of Rectal Cancer Surgery (GRECCAR). A multicentre randomised controlled trial to evaluate the efficacy, morbidity and functional outcome of endoscopic transanal proctectomy versus laparoscopic proctectomy for low-lying rectal cancer (ETAP-GRECCAR 11 TRIAL): rationale and design. BMC Cancer. 2017;17(1):253.

49. Serra-Aracil X, Pericay C, Golda T. Non-inferiority multicenter prospective randomized controlled study of rectal cancer T. Int J Color ectal Dis. 2018;33(2):241-249.
Cancer Management and Research

\section{Publish your work in this journal}

Cancer Management and Research is an international, peer-reviewed open access journal focusing on cancer research and the optimal use of preventative and integrated treatment interventions to achieve improved outcomes, enhanced survival and quality of life for the cancer patient. The manuscript management system is completely online and includes

\section{Dovepress}

a very quick and fair peer-review system, which is all easy to use. Visit http://www.dovepress.com/testimonials.php to read real quotes from published authors. 